\title{
Dying T lymphocytes call for the death of tumor cells
}

\author{
Yufang $\mathrm{Shi}^{1}$, Arthur I Roberts ${ }^{1}$, Erwei $\mathrm{Sun}^{2}$ \\ ${ }^{I}$ Department of Molecular Genetics, Microbiology and Immunology, University of Medicine and Dentistry of New Jersey, Robert Wood \\ Johnson Medical School, Piscataway, NJ 08854, USA; ${ }^{2}$ Institute of Transplantation Immunology, Organ Transplantation Department, \\ Zhujiang Hospital, Southern Medical University, Guangzhou, 510282, China
}

Cell Research (2006) 16:679-680. doi:10.1038/sj.cr.7310087; published online 17 August 2006

Immunotherapy that specifically targets tumor cells is the preferred approach to induce tumor regression. Over the past decade, significant progress has been made in devising various methods to direct the immune system to respond to tumor cells. The major hurdle for successful immunotherapy is to overcome immune tolerance in the tumor microenvironment. Recent clinical trials with dendritic cell-based vaccination [1,2] and CTLA4 blocking antibodies [3] have shown great promise, though complete cancer regression is not always achieved [4]. Currently, alternative strategies potentially leading to cancer eradication or regression in animal or clinical models are being enthusiastically pursued. In this issue of Cell Research, Wang et al. report a novel way to induce tumor immunity by the use of irradiated autologous T cells [5].

In their protocol, Wang et al. isolated activated T cells from C57BL/6 mouse splenocytes stimulated in vitro. These cells were then irradiated at $30 \mathrm{~Gy}$ and injected intraperitoneally into syngeneic mice every 5 days. After three injections, immune parameters of splenocytes from the recipient animals were assessed. Dramatic increases were observed in the percentage of CD3+, CD4+, CD8+ and CD62L+ cells, along with enhanced CTL and NK cytotoxicity to tumor cells. Importantly, administration of these irradiated autologous $\mathrm{T}$ cells also greatly inhibited the growth of tumors arising from subcutaneous inoculation with EL-4 T lymphoma cells. These findings reinforce the concept that the immune tolerance induced by tumors can be overcome by some strategies. Because this protocol is relatively simple, a better understanding of the mechanism involved may lead to its effective application in cancer patients.

Wang et al. believe that the mechanism of enhanced immune responses induced by the transfer of irradiated $\mathrm{T}$ cells lies in the resultant upregulation of GADD $45 \beta$ expression in T cells in the recipient animals. It has been shown that GADD $45 \beta$ is an inhibitor of activation-induced cell death in T cells, and thus its upregulation may allow T cells to live longer. Though the role of GADD $45 \beta$. was not definitively established in the present study, the authors did show that splenocytes from treated mice proliferate better in response to activation stimuli. The authors also demonstrated that the observed increase in proliferation does not result from induction of anti-T cell receptor antibodies by the irradiated $\mathrm{T}$ cells, since serum from immunized mice did not induce $\mathrm{T}$ cell proliferation. The increase in IL-12 production seen in cultures of splenocytes from treated mice also implies that dendritic cells or macrophages participate in the enhanced anti-tumor immunity. It is also highly likely that the irradiated $\mathrm{T}$ cells accumulate in mice after multiple injections and thus undergo secondary necrosis. Like necrotic cell death induced by direct physical damage, the necrosis of these cells would release their intracellular contents, thereby activating innate and adaptive immunity $[6,7]$.

Clearly, further studies are needed to reveal the exact mechanisms through which the anti-tumor effect of irradiated activated T cells is exerted and to evaluate its potential for clinical applications. Importantly, since EL-4 cells are of T cell origin, the anti-tumor effect may be restricted to $\mathrm{T}$ cell lymphoma. Therefore, this strategy needs to be evaluated in tumors derived from other cell types, especially those already established in situ. In addition, it would be equally interesting to examine whether cells other than activated autologous $\mathrm{T}$ cells can also elicit an effective anti-tumor immune response. On the other hand, although CTL and NK cells are suspected to be responsible for the tumoricidal effect, the precise identity of the responsible effector cells has yet to be determined. Another important outstanding question is how the irradiated T cells influence the tumor microenvironment and break immune tolerance to tumors. Nevertheless, while 
many questions remain unanswered, we expect that further investigation of the participating cells and molecules, from both the innate and adaptive immune systems, will advance our understanding of the immunobiology of tumors and lead to better strategies for clinical anti-tumor immunotherapy.

\section{References}

1 Timmerman JM, Czerwinski DK, Davis TA, et al. Idiotype-pulsed dendritic cell vaccination for B-cell lymphoma: clinical and immune responses in 35 patients. Blood 2002; 99:1517-1526.

2 Yu Z, Restifo NP.Cancer vaccines: progress reveals new complexities. J Clin Invest 2002; 110:289-294.

3 Peggs KS, Quezada SA, Korman AJ, Allison JP. Principles and use of anti-CTLA4 antibody in human cancer immunotherapy. Curr Opin Immunol 2006; 18:206-213.

4 Rosenberg SA, Yang JC, Restifo NP. Cancer immunotherapy: moving beyond current vaccines. Nat Med 2004; 10:909-915.

5 Wang L, Du F, Cao Q, et al. Immunization with autologous T cells enhances in vivo anti-tumor immune responses accompanied by upregulation of GADD45beta. Cell Res 2006: 16:702-712

$6 \mathrm{Xu}$ Y. DNA damage: a trigger of innate immunity but a requirement for adaptive immune homeostasis. Nat Rev Immunol 2006; 6:261270.

7 Scaffidi P, Misteli T, Bianchi ME. Release of chromatin protein HMGB1 by necrotic cells triggers inflammation. Nature 2002; 418:191195. 